\title{
Comparison Experienced and Les Experienced Academic Staffs' Beliefs on Post Graduate Stuedents' Educational Quality (PEQ): Case Study University of Guilan and Islamic Azad University, Rasht Unit, Iran
}

\author{
Dr. Abbas Sadeghi
}

Associate Professor, University of Guilan E-mail: asadeghi_2003@yahoo.com

Dr. Farhad Asghari

Assistant Professor, University of Guilan

Masoomeh Davoodi

Master of English Teaching, Teacher of Iran Language Institute, Rasht Branch

\author{
Atefeh Sadeghi \\ English Literature Student, University of Guilan
}

\section{Doi:10.5901/jesr.2013.v3n7p24}

\section{Abstract}

The present paper investigated the relationship among the level of experience of academic staffs, the beliefs they hold and the outcome of their successfulness in teaching quality. Two research questions have been rose which are concluded: whether teaching experiences affect the efficiency of teaching and whether the beliefs of academic staffs about teaching affect how well their students' satisfaction. The investigation is based on a study of the approach to PEQ, by 15 experienced and 15 less experienced academic staffs in two different Universities. All the academic staffs were involved in the postgraduate programs in the University of Guilan and Islamic Azad University in the Rasht Unit. All 15 experienced had at least 10 years of experience in teaching and the less experienced staffs had less than 5 years of experience and most of them had no formal practice. The research procedure involved semi-structured interviews with the academic staffs and questionnaire with post graduate students. Academic staffs' interviews included questions about how they planned their conceptions of PEQ the results showed that the experienced academic staffs employed an interactive and discovery approach to their teaching. Some of the questions asked in lessons were quite demanding cognitively. This should communicate two important messages to the post graduate students. The first was that they were responsible for their own teaching process and the second one was that teaching was about making sense out of interactions.

Keywords: Academic staff, education, quality, post graduate student

\section{Introduction}

Some important questions have commonly been raised about higher education quality. One of them is whether educational experiences affect the efficiency of higher education. Another is whether the beliefs of academic staffs about higher education affect how well their post graduate students' satisfaction. In this paper these two questions are discussed in related to the post graduate students the light of some recent research findings According to Jafary $M$, Osuly H (2000) the concept of quality consisted of the total characteristics of a specific product or an opportunity which cover abilities of meeting of special needs. Also, Hoveida. Sadat, A (2003) believes that educational quality related to the educational elements attitudes and abilities which help us to meet the students and educational manager specific needs and expectations and satisfactions.

The society's development depends to the education and higher education institutions performances particularly in the post graduate section. Iranmanesh and Kamerani (2005) emphasize that Higher education is a Human resource 
capital which can promote knowledge, skills and attitudes. Also, Akhavan Kazemi (2005) believes that if higher education systems in private and governmental part can affect on the society deeply, it can have most affection on the society. Then as Neve Ebrahim \& Karimi (2006) emphasized, it is necessary to think and consider the higher education quality in order to save human capital, financial resources and coordinate between educational development and effectiveness. There are different definitions for the word of quality. But there are not agreements about the specific definition.

Clark and Peterson (1986) suggested that individual's cognitive and other behaviors were guided by and made sense in relation to a personally held system of beliefs. Thus, the beliefs about the process of quality of higher education and the nature of post graduate and academics' performances are all important aspects that would affect the efficiency of them. Conversely, experienced academic staffs with considerable experiences of education would be likely to hold different beliefs from less experienced academics.

\section{Higher Education Planning}

Analysis of the interview protocols revealed marked differences in the focus and method of planning actives between experienced and less experienced academic staffs. When asked about planning in general, the focus of the novice academic staffs planning activities was apparently time and appointments. Thus, time and appointments was the object to search out from patients and to put down in notebooks or computers. This focus on time and appointments was also true for the less experienced academic staffs who did not mention the notebook or computer. He was familiar with the content so there was no need to plan. On the other hand, the focus of experienced planning was higher education process. Immediately, talked about how they would introduce and sequence a higher education process. One of the experienced academic staffs actually described the transition of the focus of planning:

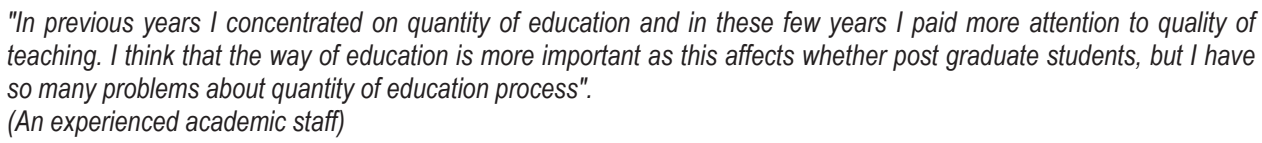

Possibly, after years of involvement on quantity aspects of education the experienced academic staffs knew by heart the subject content knowledge and had extensive schemata of their time and appointments. Consequently they could free their mind from content to focus on the best way to teaching. On the other hand, the less experienced academic staffs besides being overwhelmingly occupied with finding out about time and appointment might not have the cognitive structure for contemplating education.

The difference in focus was also apparent when discussing planning for education. The concern of the experienced when planning immediately before the teaching was again quality of education.

"I thought about the objectives of the experiment and planned some guiding questions to lead the post graduate students towards my objectives."

(An experienced academic staff)

Since experienced academic staffs used an interactive approach to education, they were more flexible in their planning of education. On the other hand, none of the less experienced academic staffs reported planning about education. Immediately before the education they still seemed preoccupied with the planning of quality of education.

A possible explanation of this difference in the focus of planning is that the two groups of dentists had different conceptions of quality of education. Consequently, the two groups of academic staffs may have different foci in their planning activities. The following sections are intended to establish a relationship between academic staffs planning of education as well as conception of quality of learning.

\section{Conceptions of higher education}

The academic staffs were asked directly about their concept of higher education and the effect of their concept on their planning. The following is based on the analysis of their interview protocols.

The less experienced academic staffs apparently viewed higher education as a collection of knowledge or facts that could be used to explain phenomena or solve post graduate students' problems. Compared to the different conceptions of higher education identified in a study of preserves academic staffs in the conception of the less 
experienced in the current study was similar to the less experienced academic staffs' conception stated as Aguirre Haggerty \& Linder (1990) emphasized that in viewpoints of Less experienced academics staffs' conception of science, science as a body of knowledge consisting of a collection of observation and explanations of how and why certain phenomena function in the universe.

This conception of higher education compared to others listed in Aguirre et al's paper lacks reference to the experimental base and the tentative nature of scientific knowledge. Less experienced academic staffs believed that higher education was a process to acquire knowledge useful for explaining phenomena and solving problems. The following quotations illustrate such a conception:

\author{
(What do you mean by "higher education?) \\ Higher education has a reason behind it. The education in higher part must take the student in the higher knowledge, \\ higher skill and higher behavior. So, we should try to explain it more and more. (A less experienced academic staff)
}

It means that education must not stop to some knowledge for examination, but the knowledge for the life. (A less experienced academic staff)

Less experienced academic staffs had similar referential aspects when they were talking about higher education. 5 of the less experienced academic staffs suggested that their concept of higher education did not affect how they planned their higher education yet the author believes that its influence on planning is quite obvious. It is only that these less experienced academic staffs have not recognized the influence.

None of the experienced academic staffs in the current study held such a "less experienced concept" of higher education. Rather two central components were identified among the conception of the less experienced which were absent in the conception of the less experienced academic staffs. The first was that higher education was related to society. Higher education could help post graduate students to understand the modern technological society and to adapt to the life within such society. The experienced academic staffs directly talked about this. Such as:

\footnotetext{
"Higher education should also let post graduate students think about application of higher education. Post graduate students should be aware of the application and appreciate the implication. Higher education should bring out the relationship of academic staffs with science and technology."

(An experienced academic staff)
}

In recent years, there has been much discussion among educators locally (Holbrook, 1990) and globally (UNESCO, 1986) about education and higher education and approach of learning. It seemed that the experienced academic staffs were generally more aware about this trend than the less experienced.

The second common component in the conception of the experienced academic staffs was that higher education as a way of thinking was described as logical analytical and empirical.

\footnotetext{
"Analytical power is essential. To achieve these, they have to possess certain degree of observation power and analytical power in learning which can develop in the higher education institution. In the postgraduate part these analytical activities are deeper."

(An experienced academic staff)
}

It is not easy to make explicit the implicit concepts involved in higher post graduate processes the proposed conception of higher education as logical analytical and empirical way of thinking seems to be a reasonably accurate summary of the conception expressed by the experienced academic staffs

Thus, the experienced and less experienced academic staffs treated the nature of higher education differently. The less experienced academic staffs considered it as a collection of memorizable fact while the experienced academic staffs treated it as a way of acquiring knowledge through empirical observation and critical thinking. Secondly the two groups of academic staffs held different views of the function of higher post graduate education process. The less experienced presumed the purpose of the higher education was to solve post graduate students' problems; whereas the experienced academic staffs conceived the function as a quality of higher education process.

\title{
4. Placement quality in Higher Education
}

Conceptions of quality are actually two sides of the same coin. A concept of quantity should evoke a corresponding 
concept of higher education. The academic staffs were asked in the interviews about their concepts of quantity and quality. From the analysis of the interview protocol on quantity and quality were clearly evident. The less experienced academic staffs had a quantitative view of teaching. Quantity is the intake of knowledge:

"For this level of post graduate students rely on the academic staff to provide them with knowledge. (What do you mean by that a post graduate student has satisfied about something?). It depends on the results of teaching."

(A less experienced academic staff)

"If I understood the academic staffs could solve the post graduates' problems in the exercise without resorting to copying from others colleagues or asking for help that meant I had learnt the knowledge."

(A Less experienced academic staff)

"That is the post graduate student understands and can solve their problems in education."

(A less experienced academic staff)

"Understanding means an ability to solve the more difficult educational problems" (A Less experienced academic staff)

Although six of the fifteen less experienced academic staffs use the words understand or understanding all of them considered understanding as an ability to solve students' problem mostly in learning rather than education. In response to the question about their conception of good education, the less experienced academic staff suggested the following:

"It means the fulfillment of the duties. The skills that are required should be covered in the educational process. The resistant students should be overseen." (Less experienced academic staff)

"Unlike that students just listen to a academic staff or even forget about what the academic staff says. If students forget what the academic staff says, the higher education is a failure." (Less experienced academic staff)

Most of the less experienced academic staffs considered higher education as the provisional duty. The experienced academic staff appeared to have deeper conception of education than the less experienced academic staffs although there was some variation. Their views of teaching were exemplified:

"Firstly if students have satisfied about something in their learning, it is reflected in their results for both in and out of the class. Secondly students' satisfaction may not be immediately noticeable. The second aspect is very difficult to measure. It relates to how well the students accept the educational process and the specific academic staff. It depends on how much satisfaction they get and what attitude they have." (Experienced academic staff)

"I do not believe provisional education can measure students' satisfaction. The important issue at least for education process is that students can say something about their continues education."(Experienced academic staff)

From the analysis of the protocols, the less experienced academic staffs were found to have a qualitative view of education. They stressed the importance of education as interpreting and applying the skill gained. One the other hand, some experienced academic staffs accepted that teaching had to be validated by scientific standards but immediately commented on the inadequacy of limiting such standards:

"There must be some interaction in the process. The academic staffs should not be too dominant. The academic staff should not direct the students but guide them. The academic staff should develop post graduate students' attitude and interest towards education. These are elements of good education. Furthermore, scientific standards are also important attribute of good teaching. But the medical organization must be sensitive about the standards inside the class." (Experienced academic staff)

"Basically the academic staff should be able to cover the standards by him/her. At a higher level, the academic staffs can help students to acquire scientific minds. At an even higher level education should involve the moral aspect." (Experienced academic staff)

The entire experienced academic staff in the current study delineated more than one level or aspect of concepts about good academic staff. Their conceptions compared with the vague concept of keeping students interested were richer and deeper. Less experienced academic staff was clearest about it. He expressed the concept of academic as a guide without being too dominant and the importance of academic staff and student interaction as an aspect of 
education. Other experienced academic staff referred to teaching as helping students to acquire scientific minds or accentuated discovering experiments and questioning in education. Most experienced academic staff also embodied the view of education as a means to bring about attitude change. All these were consistent with the qualitative or constructivist view.

While the experienced academic staff might also have a quantitative or institutional view mentioned ideas like covering the standards, they had predominantly the qualitative / constructivist view of education.

\section{Post graduate students' learning}

The following hypothesis is put forward to explain how the experienced academic staff has become more qualitatively oriented than the less experienced. Gradually, through years of experience, the academic staffs become more and more aware of the strength and difficulties students' learning (Borko \& Livingston 1989) finding the quantitative view of teaching and learning to be inadequate to account for why students understand or not. One experienced academic staff discovered that providing information and having skills were not adequate to ensure teaching. At the same time, academic staffs feel progressively more confident to have interaction with students and find that the interactive approach to teaching is more effective.

\section{Relationship between learning and education}

The quantitative orientated academic staffs held the view of education as provisional education and thus the selection and the presentation of the process were of great importance. Consequently, they equated planning and selection of skill. Furthermore, they would find the actual skill for delivery an important source of their education. They would also find the list of objectives which delineated the skills in terms of education activities or target post graduate students' behaviors were of little use to formulate what to treat the students in the class. Since, their main purpose was to provisional learning just as someone deposited money in to a short term bank account (Freire 1972) direct education and would be most appropriate. Within such an approach experiments were of little use, if of any use at all. Finally, once the provisional learning was carried out the quantitatively orientated academic staffs considered they had done their job; they have no need to reflect on how to improve their education for the next time.

On the other hand, the qualitatively- orientated academic staff perceived their role as supporting student learning through interaction and their students were the ones who really constructed their own knowledge (Wheatley, 1991). Only the interactive approach was consistent with such a qualitatively orientated viewpoint. In order for the students to have something to base the construction of knowledge on experiments were needed and formed an integral part of the education activities. In order to be functional in such an interactive class environment, the academic staffs should be able to improvise and class environment the academics should be able to improvise and be flexible (Yinger, 1989). To cope with this, the academic staffs required a clear image about the direction of the education and the type of activities that would scaffold learning. The former image required references to the list of objectives while the latter references to the skill guide. In order to provide the best assistance for students in their learning, the qualitative orientated academic staffs could reflect on the previous experiences to determine how the current education should go.

Thus, there was a definite distinction between the conception of education and learning of the experienced and less experienced academic staffs. When the dentists were classified as quantitatively orientated or qualitatively orientated all four experienced were considered as qualitatively orientated while all four novices were quantitatively orientated.

\section{Students Perceptions on quality of Education}

In order to compare the effect of academic staffs on post graduate students, a questionnaire was designed to measure some aspects of post graduate student learning. The first part of the questionnaire was aimed to measure the level of processing these students used in learning. The second was a measure of the perceived class environment in modified from Hattie and Watkins (1988). The final part was a short test about the education and learning. Two questions were set in this final part. One of the questions concerned the importance of the "motivation" concept while the other one was a problem on constraint with more data given than required. Post graduate students' answers for this final part were graded

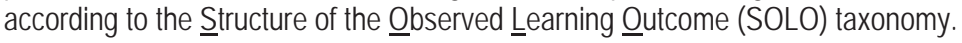




\subsection{Perception of class environment}

The difference between post gradate students in the perceived class environment was clear. The scores for the second part of the questionnaire indicated that class environment was perceived as better in the views of the post graduate students of the experienced academic staffs than those of the less experienced (ANOVA, $P<0.05$ ). The post graduate students of the experienced academic staffs generally felt happier about their class environment about their like hood of success in that environment about their impression of fair education $t$ by the academic staffs as well as about the usefulness of the class arrangement.

\subsection{Learning Approaches}

The post graduate students of the experienced academic staffs scored higher (ANOVA, $\mathrm{P}<0.05$ ) on the questionnaire items concerning deep learning than the post graduate students of the less experienced academic staffs. In other words there was some evidence that the students of the experienced academic staffs utilized a deep learning. This indicated a tendency for the post graduate students of the experienced academic staffs towards a combination of more intrinsic motivation and more concern about understanding when education.

\subsection{Learning outcome}

The data also indicated that the post graduate students of the education by experienced academic staffs were significantly (ANOVA P $<0.05$ ) higher than the other groups. These post graduate students of the experienced academic staffs were better in suggesting the importance of the concepts as well as in understanding.

\section{Discussion and Conclusion}

The current research found that the experienced academic staffs employed an interactive and discovery approach to the post graduate education. Some of the questions asked in lessons were quite demanding cognitively. This should communicate two important messages to the post graduate students. The first was that they were responsible for their own learning process. The second was that teaching was about making sense out of interactions. From this point of view the experienced academic staffs with a qualitative orientation to post graduate education imparted a more qualitative view of post graduate education to their students. This qualitative perspective then influenced the students to use a deep approach in learning. Firstly a meaning of "finding orientation" was likely to elicit a deep approach of learning (Matron 1988). Secondly the post graduate student acceptance of their responsibility for learning would also encourage a deep approach to it (Biggs 1990).

Moreover, the qualitative orientation of learning also influenced the post graduate students' perception of their class environment (Entwistle, Kozeki, \& Tait, 1989). The stress on discovery and interaction would likely increase the positive attitudes towards the class environment including their feeling while learning and their sentiment towards the academic staffs. The tendency toward accepting responsibility for their learning i. e emphasizing one's own effort would likely increases optimism about success in education (Ames \& Ames 1984).

Last but not least, a qualitative orientation to post graduate education would likely bring about better quality of learning outcomes (Matron \& Saljo, 1984; Watkins 1983). Thus noting the more qualitative orientation of learning and deeper approach with students of the experienced academic staffs it should not be astonishing to find that the achievement of these post graduate students according to the SOLO taxonomy was higher.

In conclusion, the current study suggests that the differences between the experienced and less experienced academic staffs as manifested in their learning planning conceptions, cognitive structure and conduct of class have an effect on the learning of the post graduate students. Such influence is appropriately interpreted through a framework of differences in the quantitative / quantitative orientation of post graduate education among post graduate students. If such a situation is true for academic staffs in general the importance of academic staffs carefully evaluating their own beliefs cannot be overemphasized.

\section{References}

Aguirre. M. Haggerty, S. M. \& Linder, C. J. (1990). Student-teachers conceptions of science teaching and learning: A case study in preservice science education. International journal of science education 12(4) 381-390. 
Akhavan Kazemi, M. (2005); Higher Education and Stable Political Development; Institute for Research and Planning in Higher Education,Vol. 1, pp. 13-32.

Ames, C. \& Ames R. (1984). System of student and teacher motivation: toward a qualitative definition. Journal of Educational Psychology 78(4) 535-556.

Biggs, J. B. (1990). Teaching: design for learning. Keynote discussion paper annual conference higher education research and development society of Australasia Griffith university brisance.

Borko, H. \& Promise and prospect. Science education 75, 1-8.

Hattie, J. \& Watkins, D. (1988). Preferred classroom environment and approach to learning. British journal of educational psychology, $58,345-349$.

Hoveida. Siadat A (2003) (Survey of amount of Total Quality of Management Indexes In Isfahan University. Isfahan. University of Isfahan Research vice Chancellor Branch.

Holbrook, J. B. (1990). Science education in Hong Kong: achievements and determinants. Hong Kong university of Hong Kong faculty of education. Education paper 6.

Iranmanesh, M. and E. Kamrani (2005); The Role of Higher Education in Stable Development; Institute for Research and Planning in Higher Education, Vol. 1, pp. 33- 54.

Karimi (2006); "A Study of Relationship between Triple Skills of Department Chairs and Improvement of Educational Quality"; Quarterly Journal of Research and Planning in Higher Education, Spring, Vol. 12, No. 39, pp. 61-78.

Matron, F. \& Saljo, R. (1984). Approaches to learning. In F. Matron, D. Hounsell \& N. Entwistle (Ed) the experience of learning. Edinburgh: Scottish academic press.

Jafary M, Osuly H (2000) Total Quality of Management. Irasa Culture Institution. Tehran. Iran.

Matron, F. (1988). Describing and improving learning. In R.R. Schmeck (Ed) learning strategies and learning style. New York: Plenum press, p.53-81.

Naveh Ebrahim, A. and V. Karimi (2006); "A Study of Relationship between Triple Skills of Department Chairs and Improvement of Educational Quality"; Quarterly Journal of Research and Planning in Higher Education, Spring, Vol. 12, No. 39, pp. 61-78.

Tobin, K. \& Fraser, B. (1988). Investigations of exemplary practice in high school science and mathematics. Australian journal of education 32, 75-94.

Tobin, K. Spinet, M, Byrd, S. E. \& Adams, D. (1988). Alternative perspectives of effective science teaching. Science Education, 72,433451.

UNESCO (1986). The place of science and technology in school curricula: a global survey. Paris: UNESCO.

Watkins, D. A. (1983). Depth of processing and the quality of learning outcomes. Instructional Science, 12, 49-58.

Wheatley, H. G. (1991). Constructivist perspectives on science and mathematics learning. Science Education, 75, 9-21.

Yinger, R. J. (1989). The improvisational nature of teaching. Paper presented in the sixth conference of the Hong Kong educational research association Hong Kong. 